\title{
Current Issues
}

\section{The place of Physiological Psychology in Neuroscience}

\author{
DUANE SHUTTLESWORTH \\ Kennesaw College, Marietta, Georgia \\ DARRYL NEILL \\ Emory University, Atlanta, Georgia \\ and \\ PAUL ELLEN \\ Georgia State University, Atlanta, Georgia
}

\begin{abstract}
The development of Neuroscience has raised questions about the place of Physiological Psychology in both Psychology and Neuroscience. The present paper addresses the identity crisis of Physiological Psychology by focusing on the concept of the localization of function in the explanation of brain-behavior relationships. The physiological psychologist, dependent upon the reductionistic assumption that behavior can be explained by reduction to some brain event, and the notion that we have a firm understanding of behavioral events and processes, has turned to Neuroscience for both academic identity and research sustenance. But Neuroscience lacks a molar framework, and the consequence of the flight into Neuroscience has been the deterrence of integrative theorizing about brain-behavior relationships. Only through a return to the basic intellectual tradition of our discipline can we negate this trend. By attempting to identify and understand the natural fracture lines of complex adaptive behavioral functions, physiological psychologists can begin to develop the integrative theories that will foster an understanding of brain-behavioral relationships. Doing so has significant implications for what we teach, as well as for the role we play in the Psychology-Neuroscience endeavor of the future.
\end{abstract}

In the last decade we have seen the gradual emergence of a new discipline, Neuroscience. The development of neuroscience as an academic and research entity has led to the appearance of a new scientific society, The Society for Neuroscience, with a membership roster of nearly 8,000 individuals in the United States and 43 other countries. In recent years we have witnessed the proliferation of new journals and textbooks bearing the title of "neuroscience." Those

This article is based upon papers presented as part of a symposium entitled "A question of identity: Physiological psychology's place in neuroscience," which was held during the 1983 annual meeting of the Southeastern Psychological Association in Atlanta, Georgia. Symposium participants and the titles of their presentations were: D. Shuttlesworth, "Issues underlying the identity crisis within physiological psychology"; D. Neill, "Physiological psychology's place in neuroscience"; P. Ellen, "Physiological psychology: Alive and well within psychology"; and R. Levitt, "Physiological psychology and clinical neuropsychology." Discussants were James Kalat and Walter Isaac. Reprint requests may be addressed to any of the three authors. Addresses are: Duane E. Shuttlesworth, Department of Psychology, Kennesaw College, Marietta, Georgia 30061; Paul Ellen, Department of Psychology, Georgia State University, Atlanta, Georgia 30303; Darryl Neill, Department of Psychology, Emory University, Atlanta, Georgia 30322. written by psychologists generally are called "behavioral" or "functional neuroscience" or some other phrase to reflect the nonstatic, dynamic, and functional approach to the study of the relationship between the nervous system and behavior. In fact, one of Psychology's most prestigious journals, The Journal of Comparative and Physiological Psychology, has recently divided into the Journal of Comparative Psychology, on the one hand, and Behavioral Neuroscience, on the other. All of these events point to the conclusion that the subject matter and methodology of what had previously been the province of Physiological Psychology is rapidly becoming considered to be an integral aspect of the broader discipline of Neuroscience. Such developments question the identity of Physiological Psychology as a psychological as opposed to neuroscientific endeavor.

We feel that physiological psychologists have two choices by which to respond to these developments. They can passively watch the absorption of Physiological Psychology into various of the specialties of Neuroscience (e.g., psychopharmacology, psychoendocrinology, etc.), or they can actively work to create a new definition of Physiological Psychology- 
one which will allow the discipline to remain an entity in its own right, yet still permit it to remain in close alliance with Neuroscience. If we remain passive observers, Physiological Psychology may become an "Introduction to Neuroscience" at the undergraduate level and thus will likely evolve into a teaching rather than research endeavor. At the graduate level, and at those undergraduate institutions with medical schools or biology or anthropology departments with a neuroscience component, nonpsychology departments will progressively invade the teaching domain of Physiological Psychology. The result of such passivity will be the disappearance of Physiological Psychology as an entity with any academic weight.

The following presents, in our view, a statement that can provide the basis for an understanding of the current state of affairs in which Physiological Psychology finds itself vis-à-vis Neuroscience. More importantly, it represents a positive approach to the challenge facing our discipline by indicating what may become the new role of the physiological psychologist in the scheme of Neuroscience. Our discussion delineates the nature of this new role, and suggests some of the effects it will have, not only upon what the physiological psychologist does, but also upon the future development of graduate training in our area.

\section{The Nature of the Problem}

The identity crisis confronting modern Physiological Psychology has been brewing ever since Gall and Spurzheim (Boring, 1949) conceived of the notion of the cortical localization of discrete faculties of mind. Unfortunately, the crisis is exacerbated by statements such as the one recently made by Kandel $(1981$, p. 11) that "all behavior including higher (cognitive as well as affective) mental functions is localizable to specific regions or constellations of regions within the brain. The role of descriptive neuroanatomy is therefore to provide us with a functional guide to localization within the three-dimensional neural space-a map for behavior. On the basis of this map, we can use the patient's performance as elicited in a clinical examination, to infer where the difficulties are located." This statement reflects the view concerning behavior held by most medically or biologically oriented neuroscientists.

Part of the problem of contemporary Physiological Psychology is that behavior is something simply taken for granted. In fact, even physiological psychologists have tended to by-pass and avoid the sticky conceptual problems involved in the analysis of behavior. Either they utilize those behavioral technologies that are currently in vogue, regardless of whether such technologies are indeed sensitive to the particular problem, or they concentrate their energies on mastering the latest techniques developed by colleagues in neurobiology or neurochemistry. As an example of the former, we take note of the almost compulsive application of schedules of reinforcement to the behavioral analysis of drug effects despite repeated demonstration that such techniques frequently fail to differentiate between different classes of compounds. With regard to the latter alternative, the physiological psychologist devotes much energy to the mastering of the most esoteric techniques of neurochemical assay, receptor binding, and histochemical tracing.

Unfortunately for the physiological psychologist, purely descriptive neurobehavioral studies in 1984 are no more adequate than was the purely descriptive biology of the 19th Century, and high-technology methods are only as good as the conceptual thinking behind their use. For many physiological psychologists, these different resolutions to the problem of behavioral analysis have resulted in their functional avoidance of the realm of behavior and the consequent retardation of the development of conceptual schema for relating behavior to brain function. The pursuit of neuroscience technology has become the end rather than the means to the end, and the analysis of behavioral change is being used merely as an assay for neural status.

The neglect of theory by physiological psychologists is recognized by nonbehavioral neuroscientists and further fosters the impression that there is not much to behavioral analysis since even its own practitioners tend to avoid it. The attitude held by nonbehavioral neuroscientists that "behavior is easyeverybody knows about behavior and can analyze it" is thus reinforced. It becomes possible for a neuroanatomist, for example, to blithely assert that he or she is studying the neural mechanisms of feeding behavior simply because lesions in a particular part of the brain alter food intake.

Moreover, since physiological psychologists have avoided conceptual development and have concentrated their energies on cranking out scores of experiments, they have made it appear that the causal basis of behavior lies entirely within the operationally defined independent variables of their studies, that is, the brain manipulations themselves. For example, a change in behavior resulting from a lesion may be interpreted as indicating that activity in that brain site is the cause of that behavior. Such a simplistic view ignores the complexities of both brain and behavior. By their venture into the world of Neuroscience, physiological psychologists may have gained increased precision and control of the various experimental variables they use, but they have done so at the expense of their ability to do the kinds of work that are productive of useful integrative theories of behavior. That change in direction has been unfortunate because neuroscientists have only very rarely exhibited an interest in that subject, and have often presumed that the science of psychology is really nothing more than common sense. It isn't, and the 
fact that physiological psychologists have not been attending to its problems has resulted in a virtual arrest of progress in neuropsychological thought.

\section{The Causes of the Problem}

Two assumptions implicit in Kandel's statement underlie the identity crisis of the physiological psychologist. We intend to show that both of these assumptions are detrimental for the proper functioning of the physiological psychologist. First, there is the naive reductionistic assumption that behavioral phenomena can and will simply be explained by reduction to some brain event or events, whatever their specific nature at some future time might be. Second, there is the assumption that we, indeed, have an understanding of behavior and that the problem for the physiological psychologist is simply one of mapping various behaviors onto the brain. Let us turn to the first assumption.

Reductionistic biases cause psychologists to analyze the brain, neurophysiologists to probe at the membrane level, and pharmacologists to get "high" on receptors. In point of fact, regardless of the specialty, researchers are more likely to read the literature pertaining to phenomena below the level of their research than those above that level (Neill, 1983). As a consequence, neuroscientists of a cellular/molecular orientation are not familiar with the behavioral literature. And, in regards to physiological psychologists, we resort to literature in the cellular/molecular areas for our explanatory schemata rather than the behavioral literature.

Current trends in description and explanation in modern Physiological Psychology imply that biological phenomena are simpler units for analysis than are behavioral ones. The resultant outcome has been the development of minitheories about the operation of specific aspects of brain physiology and function, and the consequent deterrence of the development of integrative and generalizable theories of behavior. Physiological psychologists know that while the behaviors they study are produced by the activities of thousands, if not millions, of neurons, they still do not know the functional plan of the mammalian brain, and that cellular/molecular studies do not necessarily help explain the large-scale neural phenomena that underlie behavior. Thus, there is the oft-expressed hope that someday in the future, someone will provide the conceptual umbrella under which the phenomena we are studying piecemeal can be effectively related.

Because of our reductionistic bias, we often make the assumption that behavior is a unitary phenomenon requiring no further analysis. As a consequence, we assume that once the details of brain function are delineated, the problem of behavioral or psychological function will be readily resolved. Given this perspective, it is not unreasonable that physiological psychologists have become neuroscientists concentrating on Neuroscience qua Neuroscience rather than behavior. Unlike neurophysiological phenomena which are often reducible to the activity of specific brain elements, behavioral or psychological functions are not unitary events requiring no further analysis. To the extent that we accept a reductionistic model, we thus have denied the validity of our own enterprise in the study of behavior. Unfortunately, behavior is not a "thing" in its own right to be simply mapped into brain structure; rather, behavior is a complex process requiring as much analysis as any neurophysiological phenomenon.

Let us now return to the second assumptionnamely that we know something about psychological functions-and examine this notion in some detail. What is a psychological or behavioral function and how is one identified? To gain some insight into this issue, we would like to use the lesion approach to the study of brain function as an example. The lesion approach tends to impute to a structure those functions which are lacking when organisms sustain damage to that structure. For example, if performance on a task requires that an animal inhibit or withhold a response following damage to a brain structure such as the septal or hippocampal area, it is not uncommon to assume that there indeed is a real behavioral or response inhibitory function that is a property of the damaged tissue. Gregory (1961) warned about the kinds of errors attendent to making inferences of this kind. He pointed out how misleading it would be to infer a squeak suppression function to a missing part of a radio if the radio squeaked every time that part was removed. There are several flaws with this type of approach to the identification of function. First, the functions imputed to be missing as a result of damage may actually reflect only the biases of the experimenter in choosing a particular task to use as the behavioral assessment tool. Had another task been chosen, another behavioral function would have been imputed to the damaged structure. James Olds (1959) once commented upon the fact that the hippocampus changes function with each new experiment. Secondly, the circularity of the reasoning process underlying this approach to the study of brain function is clearly apparent.

Furthermore, this approach neglects the very real possibility that the sequelae of brain damage do not necessarily reveal anything about the functions of the involved tissue. While the distinction between symptoms and functions has been made since J. Hughlings Jackson's time, it has been obscured by the rash of neurobehavioral research seeking to uncover the function or functions of one or more structures of the brain. Localization of symptoms is one thing; localization of functions is another. Additionally, and by now it is almost a truism, there is the fact that what is measured following brain damage is not the 
function of the damaged tissue but rather the adaptation of the remaining tissue to the demands of the behavioral task being used to assess the damage. This point was made many years ago by Kurt Goldstein (1939), who suggested that we should interpret symptoms of brain damage in the context of the organism's total adaptation rather than simply descriptively as revealing lost functions.

Most neurobehavioral studies generally define a function in terms of either the experimental variables being manipulated or the particular behaviors studied in a given task. The functions so defined are conceptually empty and are nothing more than reifications of operationally defined variables or tasks. To this extent, the neurobehavioral endeavor has not proceeded far beyond the level of sophistication exemplified in Gall and Spurzheim's phrenology. Furthermore, it is quite evident from this analysis that we do not have the grasp on behavior that our neuroscience colleagues believe. In fact, it would appear from this examination that our understanding of behavioral phenomena does not exceed that of the nonpsychologically trained layman.

Given the examination of basic assumptions made by physiological psychologists as they attempt to localize various behavioral or psychological functions to specific brain locations, it is readily apparent that the identity crisis faced by them is really of their own making. It derives directly from the kinds of assumptions made, and, as we have suggested, such assumptions are neither warranted nor necessary for the proper pursuit of the ends of the discipline.

\section{A Possible Resolution}

Luria (1966) has provided us with a methodological alternative by emphasizing the different meanings that can be ascribed to the concept of function. Function can refer to the work performed by a given tissue, thus implying a certain invariance that is of little value to our discussion concerning the relationship between behavioral and neural events. As noted by Laurence and Stein (1978), this usage of the term may lead to a category confusion, as exemplified by the references to cells of the brain as feature analyzers, place detectors, response inhibitors, etc. This terminology gives the impression that indeed the brain cells are feature analyzers, place detectors, etc. Feature analysis, place detection, etc., are not brain functions. Rather, they are the consequences of brain activity.

This brings us to Luria's second usage of the term function. Luria suggests that the term refers to the complex activity of a system or an organism that produces some adaptive relationship with the environment. Here the emphasis is on the behavioral product that is achieved or observed rather than the means to that product or end. Thus, response inhibition, place detection, feature analysis, and the like are products of the organization and integration of a number of tissues and/or organ systems. In this sense, behavioral functions are adaptive consequences and accomplishments of brain activity rather than specific properties of the brain or its multifaceted parts.

It is our view that this usage of the term "function" can provide the context for the work of the modern physiological psychologist, and that the view of function as an intrinsic invariant property of a tissue or structure can provide the context for the work of the neurobiologist.

If we accept Luria's distinction in the two meanings of "function," it is apparent that a psychological or behavioral function is not something that can be simply mapped onto three-dimensional neural space as suggested by Kandel. Rather, the behavioral function is the organism's adaptation to the task and actually represents an emergent consequence of a variety of brain processes and functions.

This approach to function defines the task of Physiological Psychology. The attempt to localize functions to particular structures should no longer be a concern. Rather, there needs to be a precise specification of the various symptoms following brain damage. Such specification will identify the "natural fracture lines" of the emergent function. This will require greater precision not only in the methodology of brain-damage research, but also, perhaps more importantly, greater precision in behavioral methodology which can only come as a result of more sophisticated theory.

No longer can we be content merely with the operational definitions of psychological functions. Psychological functions will constitute the theoretical terms of neurobehavioral models. These terms must be related to more than data in the behavioral domain or they will be nothing more than conceptually empty tautologies. As suggested by Nadel and O'Keefe (1974), these terms will need anchoring to data in the neural domain. Once the terms are tied to two or more empirical domains, they can be used to generate other constructs and lead to the development of a network of terms that can be substantiated or disconfirmed at a number of levels. A prime example of this approach is to be found in O'Keefe and Nadel's (1978) book, The Hippocampus as a Cognitive Map, which reflects theorizing that is not merely a reductionism to a lower, more molecular level of analysis. Instead, it demonstrates a theoretical approach requiring the development of correspondence rules by which a theoretical term can at one and the same time be defined behaviorally as well as neurally. The physiological psychologist can become a major contributor to the development of such dictionaries. As a result, the role of Physiological Psychology will change. No longer will the physiological psychologist need to 
compete with neurobiologists for technical sophistication or eschew behavioral theory. The role of the physiological psychologist in the scheme of things will be that of the integrator and theoretician rather than the scientific practitioner.

This view has considerable implications for the future role of the physiological psychologist in both academic and applied settings. If adopted, it will have significant impact on the way we go about training our graduate students. More specifically, it will cause us to alter the traditional focus of our courses from a developing emphasis on the anatomy and physiology of the nervous system to a genuine consideration of the adaptive nature of brain function. Greater emphasis on the teaching of behavioral theory and its significance vis-à-vis Neuroscience will be required. Not only will the student physiological psychologists be alert to the latest technological developments in Neurobiology; they must also be able to comprehend their role in the broader context of the study of brain-behavior relationships. In essence, we advocate a return to the basic intellectual tradition of our discipline rather than a continuation of the current trend to become high technologists devoid of a theoretical/conceptual orientation.

In the clinical setting, the new physiological psychologist will be of genuine assistance to the practitioner concerned with the remediation of symptoms following brain damage. By viewing function in the sense of an adaptive activity rather than a fixed property of the brain, he/she can seriously entertain notions of behavioral recovery following brain damage. To the extent that the physiological psychologist can specify the conditions under which particular lesions of the brain give rise to symptoms having a particular qualitative character, the possibility for developing behavioral strategies that will restore the loss of function will become possible. In short, the physiological psychologist will become a major contributor to the practitioner's work.
It is our position that this perspective can provide the framework for the future development of Physiological Psychology. An emphasis on functional behavioral analysis makes Physiological Psychology a major partner in the neuroscientific endeavor. To quote Karl Lashley, "the study of psychological processes furnishes a mass of factual material to which the laws of nervous action in behavior must conform" (1960, p. 208).

\section{REFERENCES}

Boring, E. G. (1949). A history of experimental psychology. New York: Appleton-Century-Crofts.

Goldste IN, K. (1939). The organism. New York: American Book.

GREGORY, R. (1961). The brain as an engineering problem. In R. Thorpe \& O. Zangwill (Eds.), Current problems in animal behavior (pp. 307-330). Cambridge: Cambridge University Press.

KANDEL, E. (1981). Brain and behavior. In E. Kandel \& J. Schwartz (Eds.), Principles of neural science (p. 11). New York: ElsevierNorth Holland.

LAshley, K. (1960). Basic neural mechanisms in behavior. In F. Beach, D. Hebb, C. Morgan, \& H. Nissen (Eds.), The neuropsychology of Lashley (pp. 207-208). New York: McGraw-Hill.

Laurence, S., \& Stein, D. (1978). Recovery after brain damage and the concept of localization of function. In S. Finger (Ed.), Recovery from brain damage: Research and theory. New York: Plenum Press.

LuriA, A. (1966). Higher cortical functions in man. New York: Basic Books.

NADEL, L., \& O'KeEFe, J. (1974). The hippocampus in pieces and patches: An essay on modes of explanation in physiological psychology. In R. Bellairs \& E. Gray (Eds.), Essays on the nervous system. A festschrift for Professor J. Z. Young (pp. 367 390). Oxford: Clarendon Press.

Neill, D. (1983, March). Physiological psychology's place in neuroscience. Paper presented at the 1983 Annual Meeting of the Southeastern Psychological Association, Atlanta, Georgia.

O'KeEFE, J., \& NADEL, L. (1978). The hippocampus as a cognitive map. London: Oxford University Press.

Olds, J. (1959). High functions of the nervous system. Annual Review of Physiology, 21, 381-402.

(Manuscript received February 17, 1984; accepted for publication February 23, 1984.) 